\title{
IMPLEMENTATION OF A REAL-TIME STACKING ALGORITHM IN A PHOTOGRAMMETRIC DIGITAL CAMERA FOR UAVS
}

\author{
Ahmad Audi $^{\mathrm{a}, \mathrm{b}}$, Marc Pierrot-Deseilligny ${ }^{\mathrm{a}, \mathrm{c}}$, Christophe Meynard ${ }^{\mathrm{a}}$, Christian Thom ${ }^{\mathrm{a}}$ \\ ${ }^{a}$ IGN, LaSTIG, LOEMI, 73 Avenue de Paris, 94160 Saint-Mandé, France - firstname.name@ign.fr \\ ${ }^{\text {b } U n i v e r s i t e ́ ~ P a r i s-E s t, ~ 6-8 ~ A v e n u e ~ B l a i s e ~ P a s c a l, ~} 77420$ Champs-sur-Marne, France - audiahmad@ hotmail.com \\ ${ }^{c}$ ENSG, 6-8 Avenue Blaise Pascal, 77420 Champs-sur-Marne, France - Marc.Pierrot-Deseilligny @ ensg.eu
}

KEY WORDS: UAVs, image stacking, real-time, hardware/Software co-design, FPGA, image processing.

\begin{abstract}
:
In the recent years, unmanned aerial vehicles (UAVs) have become an interesting tool in aerial photography and photogrammetry activities. In this context, some applications (like cloudy sky surveys, narrow-spectral imagery and night-vision imagery) need a longexposure time where one of the main problems is the motion blur caused by the erratic camera movements during image acquisition. This paper describes an automatic real-time stacking algorithm which produces a high photogrammetric quality final composite image with an equivalent long-exposure time using several images acquired with short-exposure times.

Our method is inspired by feature-based image registration technique. The algorithm is implemented on the light-weight IGN camera, which has an IMU sensor and a SoC/FPGA. To obtain the correct parameters for the resampling of images, the presented method accurately estimates the geometrical relation between the first and the $N^{t h}$ image, taking into account the internal parameters and the distortion of the camera. Features are detected in the first image by the FAST detector, than homologous points on other images are obtained by template matching aided by the IMU sensors. The SoC/FPGA in the camera is used to speed up time-consuming parts of the algorithm such as features detection and images resampling in order to achieve a real-time performance as we want to write only the resulting final image to save bandwidth on the storage device. The paper includes a detailed description of the implemented algorithm, resource usage summary, resulting processing time, resulting images, as well as block diagrams of the described architecture. The resulting stacked image obtained on real surveys doesn't seem visually impaired. Timing results demonstrate that our algorithm can be used in real-time since its processing time is less than the writing time of an image in the storage device. An interesting by-product of this algorithm is the $3 \mathrm{D}$ rotation estimated by a photogrammetric method between poses, which can be used to recalibrate in real-time the gyrometers of the IMU.
\end{abstract}

\section{INTRODUCTION}

In the last decades, the civil use of unmanned aerial vehicles (UAVs) has grown exponentially, this led the LOEMI team of IGN/LaSTIG (Institut National de l'Information Géographique et Forestière) to design an ultra-light digital camera (Martin et al., 2014) better adapted for exploiting photogrammetry and metrology applications than consumer cameras when using UAVs (Daakir et al., 2016), (Tournadre et al., 2015). This SoC/FPGA-based camera will make possible to implement in hardware some realtime image processing algorithms. Night-time surveys and narrow spectral bandwith imagery are one of the next applications targeted by IGN, this type of applications needs a long-exposure time imagery that usually exhibits a motion blur due to erratic movements of the UAV. This paper presents a stacking algorithm that can provide in real-time an accurate blur-free composite image with an equivalent long exposure where only the resulting image will have to be saved in order to ensure a better overall frame rate. The main part of this algorithm is feature-based image registration (Le Moigne et al., 2010) that consists of features detection in the first image, features matching in all other images, geometrical transformation estimation (Estimation of the mapping function parameters) between all images, and resampling of the images. This paper is based on an article submitted to the Sensors journal. Actually, the UAV is submitted to rotation or translation movements in the 3D-space. So to take into account the perspective effects, our problematic needs to have the DSM (Digital Surface Model). Unfortunately, this treatment is not possible in real-time until now. In absence of the DSM, we study two particular approaches in our work:

- the first approach considers that the drone is quasi-stationary. In this situation, the optical center of the camera is fixed, which leads to take only into account only the orientation of the camera and therefore only the deformation due to $3 \mathrm{D}$ rotation is corrected.

- the second approach considers that the drone undergoes a 2D translation in addition to the $3 \mathrm{D}$ rotation. In this situation, we use the 2D planar homography as geometrical transformation between images which leads to correct results when the scene can be considered as planar.

Some tests have been achieved on a Copter 1B UAV from Wikhydro equipped with the IGN camera to assess accuracy of the method and some of the results are presented.

\section{HARDWARE}

Figure 1 and Figure 2 show the IGN ultra-light photogrametric camera in which we implement our algorithm. It is based on a global shutter CMOS 20MP sensor (CMOSIS CMV20000) and a Zynq 7030 SoC/FPGA from Xilinx consisting on a two ARM cortex A9 CPUs running a Linux OS, and a programmable logic portion used originally to acquire image data from the sensor. It also has low-cost inertial sensors that will provide information about attitude of the camera during shootings. To make accurate $3 \mathrm{D}$ geometric measurements from images, this camera is 


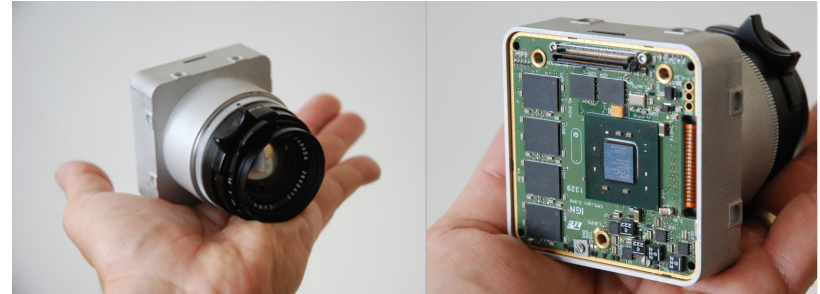

Figure 1. IGN lightweight photgrammetric camera

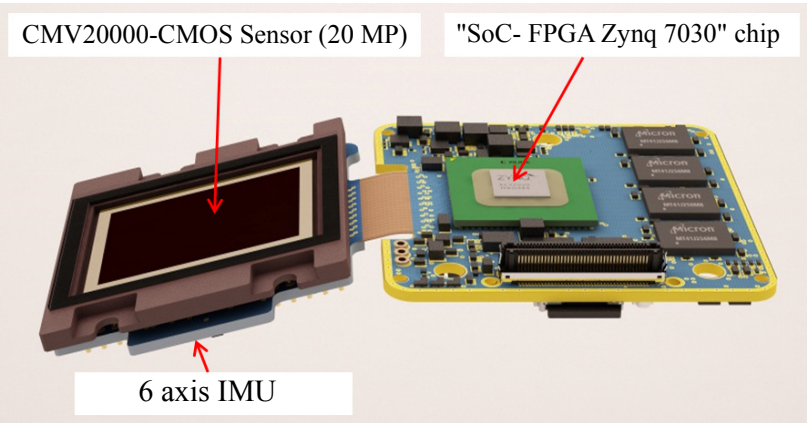

Figure 2. The CMOS Sensor with the inertial sensors and the embedded intelligence (SoC/FPGA).

calibrated (standard radial distortion model) before surveys using MicMac, the free open-source photogrammetric software developed at IGN (Pierrot Deseilligny and Clery, 2011).

\section{IMAGE AND DATA PROCESSING}

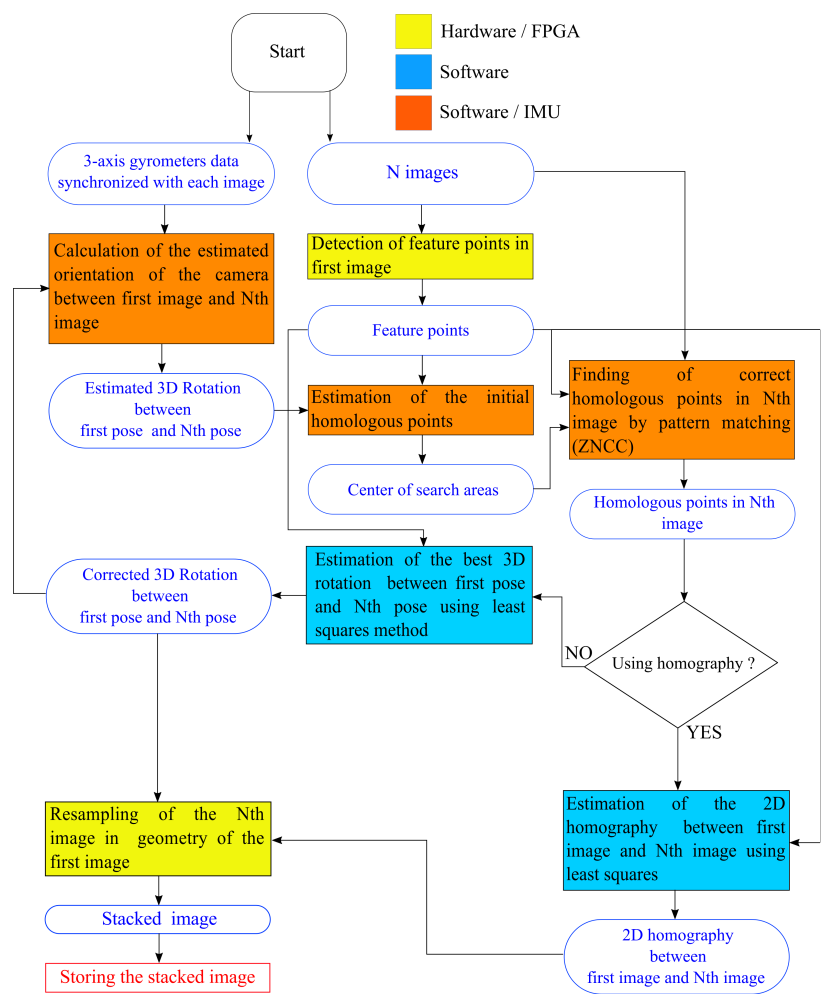

Figure 3. Architecture of the algorithm (hardware/software codesign).

This algorithm is implemented and executed in the camera. At first, it was developed on a desktop PC. The architecture of the algorithm is illustrated in Figure 3.

\subsection{FEATURES DETECTION}

In our work, the choice of the feature detection algorithm was dictated essentially by its ability to find in a single image a reasonable number of good features and by its simplicity. After analyzing main existing feature detectors (Pena, 2012) (Le and Gonzalez, 2009), we selected the FAST (features from accelerated segment test) detector algorithm developed by (Rosten and Drummond, 2005). We added a simple grid-adapted features selection consisting of partitioning the image into blocks and keeping only in each block the first feature found by the detector, thus achieving a more uniform distribution of features as shown in Figure 6. A hardware implementation of the FAST detector has been done by (Kraft et al., 2008), that are more complex and uses more resources in their design. The global design presented

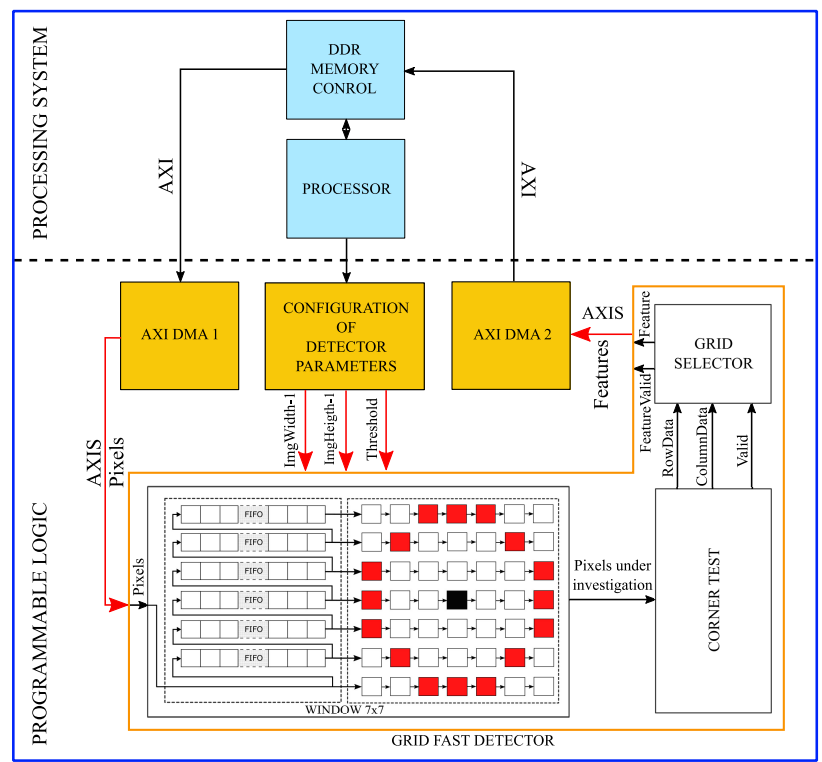

Figure 4. The global design of the grid fast detector implementation.

in Figure 4 was implemented and tested on the Xilinx/Zynq of the camera. Our solution uses the standard DDR RAM memory for storing both images and coordinates of features. The AXI bus was used to connect the processor system and memory. The AXIS transports the data stream between modules in master/slave modes. Table 1 illustrates the resources used in FPGA for the implemented design.

\begin{tabular}{|c|c|c|c|}
\hline Resource & Used & Available & $\%$ \\
\hline Slice LUTs & 232 & 78600 & 0.30 \\
\hline Slice of 4 registers & 472 & 157200 & 0.30 \\
\hline Memory (blocks of 36 KB) & 6 & 265 & 2.26 \\
\hline Clocking & 1 & 32 & 3.13 \\
\hline
\end{tabular}

Table 1. Resources usage of the FAST detector design in Xilinx/Zynq-7030 FPGA.

\subsection{FEATURES MATCHING}

Using the internal orientation parameters produced by the optical calibration process and the 3D rotations between poses provided by IMU, we can compute the predicted position of homologous points of detected features (Figure 5). We consider this predicted position as the center of a search area in which the correct position should be found by ZNCC template matching with sub-pixel accuracy. This part achieves already in software a real-time performance given the fact that useless features are filtered out by 
the grid selector and that the size of the search areas is small (11 $\times 11$ pixels) due to IMU prediction. We benefit from the presence of two cores on the camera to accelerate this part in software using the OpenMP library.

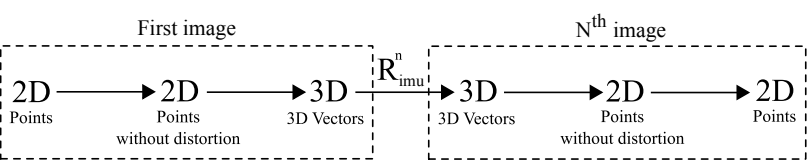

Figure 5. Successive steps to obtain the coordinates of corresponding location of pixel in other image. $R_{i m u}^{n}$ is the $3 D$ rotation obtained by IMU between the first image and the $N^{\text {th }}$ image.

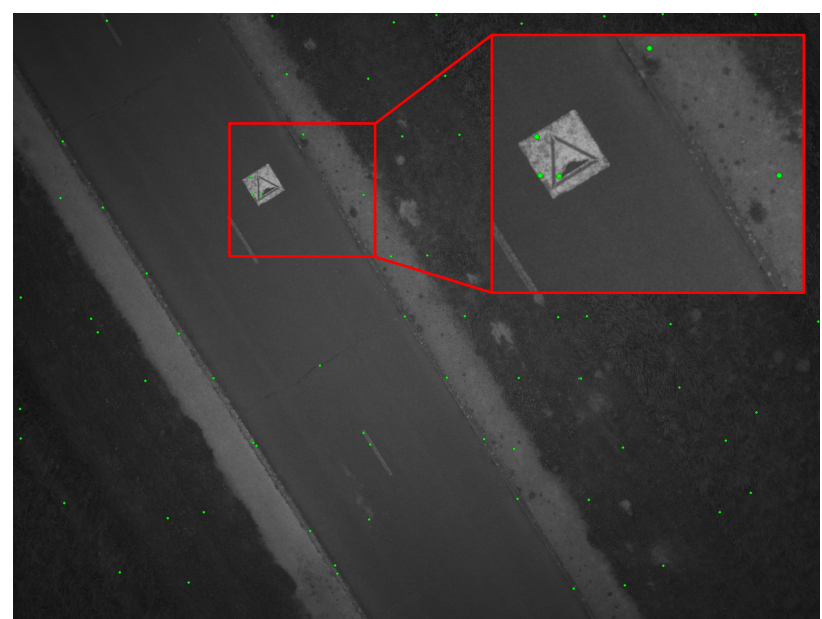

Figure 6. Features detected by FAST algorithm in the first image. The threshold choosen here is 15. The number of blocks of grid is fixed to $10 \times 10$. The resolution of image is $2560 \times 1920$ pixels.

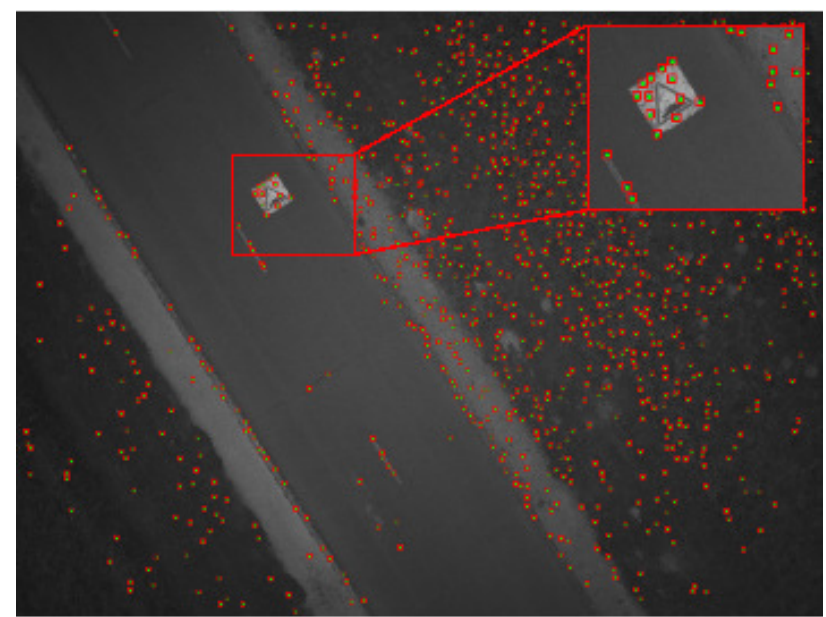

Figure 7. Search areas obtained by the gyrometers of IMU between the first image and the $10^{\text {th }}$ image. The green points represent the homologous points obtained by template matching. The size of each search area is $11 \times 11$ pixels. The resolution of image is $2560 \times 1920$ pixels. $M \times N=60 \times 60$. Threshold $=8$

\subsection{ESTIMATION OF GEOMETRICAL TRANSFORMA- TION BETWEEN IMAGES}

After having good homologous pairs between images, we need to estimate optimally the geometrical relation that permits to map pixel coordinates from one image to another. Two geometrical transformations can be used here, the 3D rotation between poses and the 2D homography between images. The choice of one of these transformations depends on the real movement of the camera as explained in the introduction. The estimation is established by least squares method. In this context, outliers are eliminated automatically to improve the robustness. This part has already in software a real-time performance.

\subsection{GEOMETRICAL TRANSFORMATION USED FOR RE- SAMPLING}

For the resampling, we use either the $3 \mathrm{D}$ rotation or the $2 \mathrm{D}$ homography. RMS errors produced by the least squares estimation serve as a quality factor to choose one of them. The internal orientation parameters (in our case: the coordinates of the centre of projection of the image, the focal length of the lens, the coordinates of the center of distortion, the radial lens distortion polynomial coefficients) produced by the optical calibration process and the estimated geometrical transformation permit to map all pixel coordinates from the first image to others: let us consider that

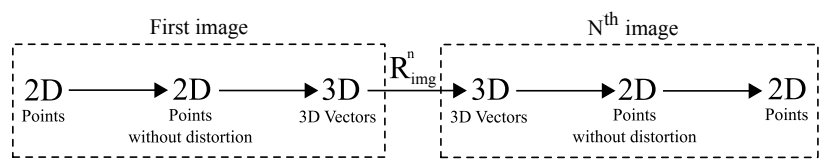

Figure 8. Successive steps to obtain the coordinates of corresponding location of pixel in other image. $R_{i m g}^{n}$ is the estimated $3 D$ rotation between the first image and the $N^{\text {th }}$ image.

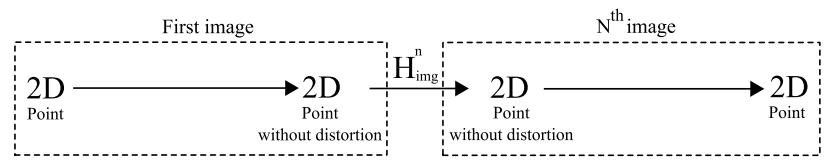

Figure 9. Successive steps to obtain the coordinates of corresponding location of pixel in other image. $H_{i m g}^{n}$ is the estimated $2 D$ homography between the first image and the $N^{\text {th }}$ image.

$P_{i}^{1}(u, v)$ is a $2 \mathrm{D}$ point in the plan of the first image. First, the geometric distortion is removed, then $P_{i}^{1}(u, v)$ is transformed to a $3 \mathrm{D}$ vector $\overrightarrow{V_{i}^{1}}(x, y, z)$ in the camera coordinates system, the origin of this system is at the optical center of the camera. After applying the inverse of the $3 \mathrm{D}$ rotation $\left(R^{n}\right)$ to $\overrightarrow{V_{i}^{1}}(x, y, z)$, we obtain a new vector $\overrightarrow{V_{i}^{r}}\left(x^{\prime}, y^{\prime}, z^{\prime}\right)$ that will be projected into the plan of the $N^{t h}$ image to have the corresponding 2D point $P_{i}^{n}\left(u^{\prime}, v^{\prime}\right)$ after applying again the geometric distortion.

\subsection{NON-ACCELERATED IMAGE TRANSFORMATION AND RESAMPLING SOLUTION}

The geometrical transformation is applied on each pixel of the first image to obtain the location of corresponding point on other images. Then, we use bilinear interpolation to find the resampled value of each pixel on other images. Removing and applying distortion is the most computation intensive task in the geometrical transformation process. Timing results presented in Table 3 demonstrate that this phase is still an important part of the timing budget, so we should accelerate it using a new approach.

\subsection{ACCELERATION IN SOFTWARE OF THE RESAM- PLING}

The mapping process of pixels of the first image in other images is expensive in time essentially due to the removing/applying the distortion in the process as presentend in Figure 8 and Figure 9 and to the bilinear interpolation. We introduce here a bilinear approximation approach consisting on dividing the first image into 
$K \times L$ blocks, and on computing the precise mapping of the only corners of each block for all other images, then other pixels inside each block can be computed using the bilinear interpolation. Thus avoiding a lot of multiplications. To accelerate the find-

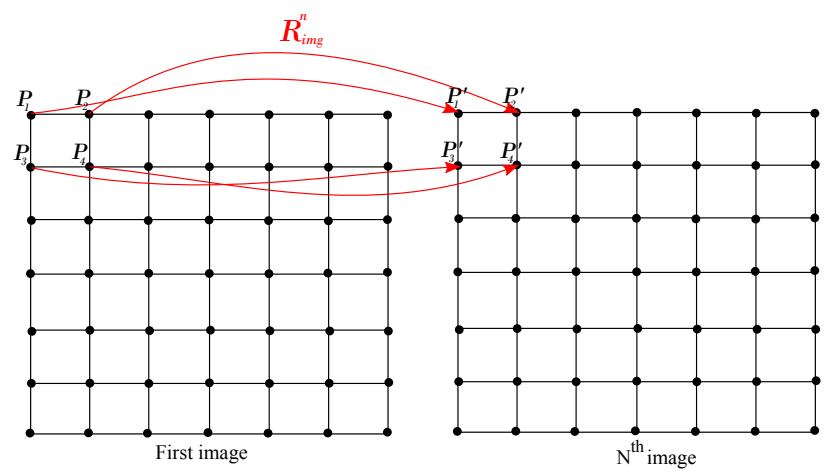

Figure 10. The accelerated approach of image transformation and resampling. $T_{i m g}^{n}=R_{i m g}^{n}$ is the geometrical transformation between the first image and the $N^{\text {th }}$ image. $p_{1}, p_{2}, p_{3}, p_{4}$, are the corners of the first block of the first image. $p_{1}{ }^{\prime}, p_{2}{ }^{\prime}, p_{3}{ }^{\prime}, p_{4}{ }^{\prime}$ are the projected points in the $N^{\text {th }}$ image. $K \times L$ is configured so that the shape of the block is always a square.

ing of the resampled value of each pixel on other images, we use the nearest neighbor interpolation instead of the bilinear interpolation. Results demonstrate that the quality of stacking image doesnt' show a major loss of accuracy as shown in Figure 21. In terms of computing, this approach provides a near real-time performance as shown in Table 3. Consequently, it should be implemented in the FPGA to obtain a real-time performance.

\subsection{IMPLEMENTATION IN HARDWARE OF THE AC- CELERATED SOFTWARE RESAMPLING}

To benefit maximally from the presence of the SoC in the camera, one of the two cores calculates the location of the four projected points for the next block in all images using the preceise mapping, the second one calculates the parameters of the " $y$ " parts of the next line inside the block. During that time, the IP core in the programmable logic part calculates the " $x$ " part of the bilinear function, process the stacked line and than writes the stacked line on the appropriate position in the memory. Since the rotation between images is considered as small, there is a big chance that projected points are located one after another on the same line. For this reason, we use a cache of 8 bytes in order to reduce the access to memory. We use the VivadoHLS software from Xilinx to design our algorithm by compiling the adapted $\mathrm{C} / \mathrm{C}++$ code into logic elements. Table 2. illustrates the resources used

\begin{tabular}{|c|c|c|c|}
\hline Resource & Used & Available & $\%$ \\
\hline Slice LUTs & 1742 & 78600 & 2.21 \\
\hline Slice of 4 registers & 2400 & 157200 & 1.52 \\
\hline $\begin{array}{c}\text { Memory } \\
\text { (blocks of 36 KB) }\end{array}$ & 2.5 & 265 & 0.94 \\
\hline DSPs & 1 & 400 & 0.25 \\
\hline
\end{tabular}

Table 2. Resources usage of the resampling design in Xilinx/Zynq-7030 FPGA.

in the FPGA for the resampling part. Our design uses little FPGA resources, that allows to duplicate it to accelerate the processing.

\section{RESULTS AND DISCUSSION}

\subsection{TEST SURVEYS}

We performed some experiments to quantify the accuracy and performance of our accelerated resampling method. We used the hardware previously described in "hardware" section. Several datasets have been acquired by the IGN camera. Each dataset consists in sequence of ten images acquired at the maximum frame rate of the CMOS (30 images/s).

For this first test, the camera prototype we used was fitted with a Bayer sensor and has a resolution of $20 \mathrm{MP}(5120 \times 3840)$. Our work aims to be used for a narrow spectral imaging system consisting of several monochrome IGN cameras, thus we use the 5 MP $(2560 \times 1920)$ grayscale images obtained from the original pixel-stream by averaging each green pair of the Bayer pattern. The Copter 1B UAV from Wikhydro used for surveys is shown in Figure 11

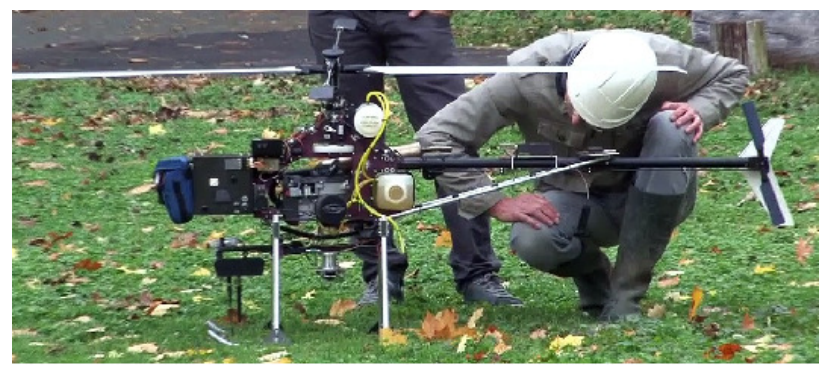

Figure 11. UAV type Copter 1B developed by Wikhydro. Source: (Website wikhydro)

\subsection{TIMING RESULTS (IN SECONDS)}

\begin{tabular}{|c|c|c|c|c|c|}
\hline & $\begin{array}{c}\text { Features } \\
\text { detection } \\
(650 \\
\text { points) }\end{array}$ & $\begin{array}{c}\text { Matching } \\
\& \\
\text { Estimation } \\
\text { of 3D } \\
\text { rotation }\end{array}$ & \multicolumn{3}{|c|}{ Resampling } \\
\hline \multicolumn{7}{|c|}{} & 0.01 & 4.72 & 0.94 & 0.66 \\
\hline $\begin{array}{c}\text { PC } \\
\text { (Soft) }\end{array}$ & 0.21 & 0.005 & 2.06 & 0.42 & 0.38 \\
\hline $\begin{array}{c}\text { PC (Soft) } \\
2 \text { Threads }\end{array}$ & 1.64 & 0.83 & 36.94 & 7.24 & 5.28 \\
\hline $\begin{array}{c}\text { Camera } \\
\text { (Soft) }\end{array}$ & 0.42 & 18.48 & 3.81 & 2.65 \\
\hline $\begin{array}{c}\text { Camera } \\
\text { (Soft) } \\
2 \text { Threads }\end{array}$ & 0.02 & & & & \\
\hline $\begin{array}{c}\text { Camera } \\
\text { (Hard) }\end{array}$ & & & & \\
\hline $\begin{array}{c}\text { Camera } \\
\text { (Hard) } \\
\text { 4 IPs }\end{array}$ & & & & & \\
\hline
\end{tabular}

Table 3. (1): geometrical transformation using the $3 D$ rotation and bilinear interpolation, (2): Accelerated geometrical transformation with bilinear function method and bilinear interpolation, (3): Accelerated geometrical transformation with bilinear function method and Nearest-neighbor interpolation All tests were run on two platforms: Firstly, a HPZ210 Workstation with an Intel Xeon $3.30 \mathrm{GHz}$ processor and $8.0 \mathrm{~GB}$ of RAM. Secondly, the photogrametric IGN camera with $666 \mathrm{MHz}$ dualcore ARM Cortex-A9 processors. The number of images is 10. 
Table 3 shows that for software versions, the factor gain in time is 5 between (1) and (2) and 7 between (1) and (3). This factor becomes 8 between the initial software version(1) and the hardware version. Benefiting from the presence of dual-core processors in the camera, we use the OpenMP library to accelerate the algorithm, the number of threads is set to 2 , the time of processing of this part is reduced by half. Our method achieve a real-time performance in comparison to the time needed for writing an image in the SD-Card storage. The IP duplication is used to process in

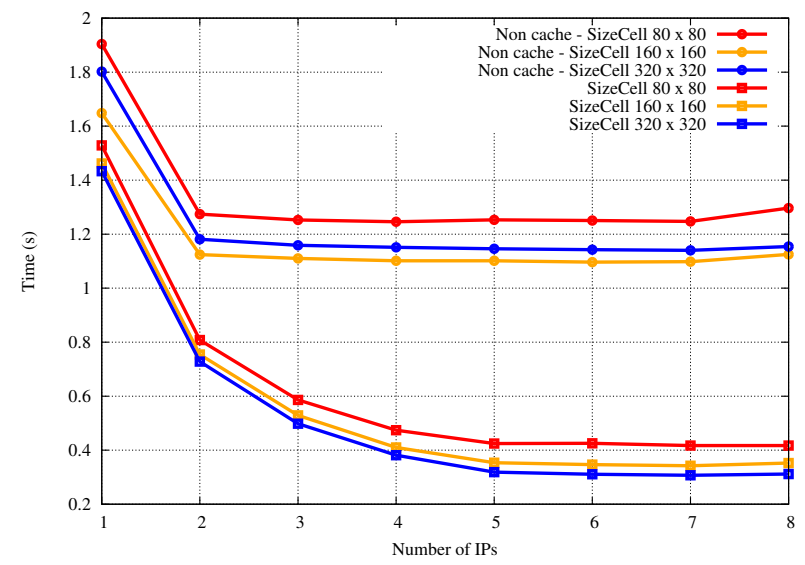

Figure 12. Time of resampling part in function of number of hardware IPs used.

parallel several lines of each block. (Figure 12) shows the effect of the impact of the number used IPs with respect to the size of the cells of the grid and the use of the eight pixels input cache. So we choose to use 8 IPs in the following tests, thus gaining a factor of 5 in computing time.

\subsection{OUALITY OF THE ORIGINAL STACKED IMAGE}

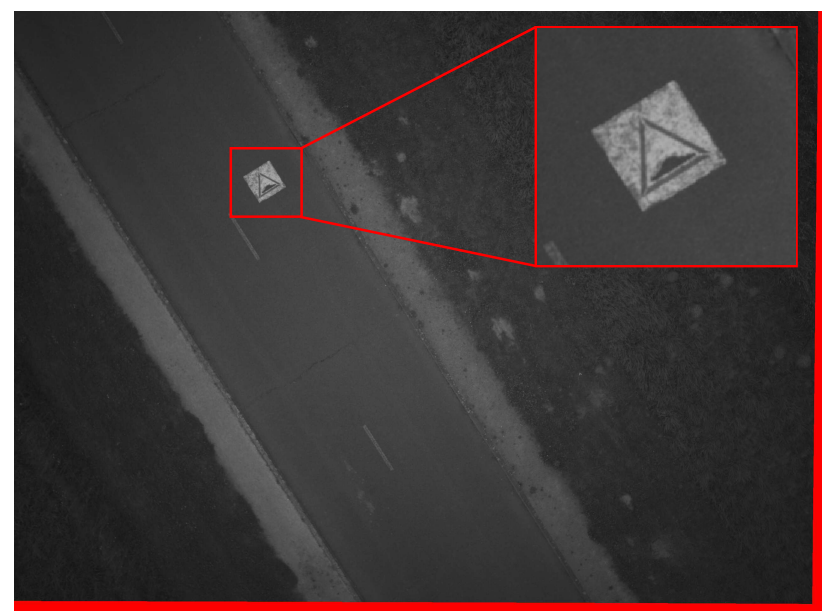

Figure 13. The stacked image. The red part is the non-commun part between the first image and the $10^{\text {th }}$ image.

Figure 13 demonstrates visually that no motion blur affect the stacked image. By comparing the stacked image with the average image as shown in Figure 14, we can constate that our method can be used to eliminate the motion blur caused by erratic movement of the camera.

To qualify more precisely the quality of the stacked image, we calculate respectively the radiometric profile of the same line of both the stacked image and the first image. Figure 15 presents that the two profiles are very close each to other. The radiometric profile of the stacked image is more smooth due to the geometrical interpolation.

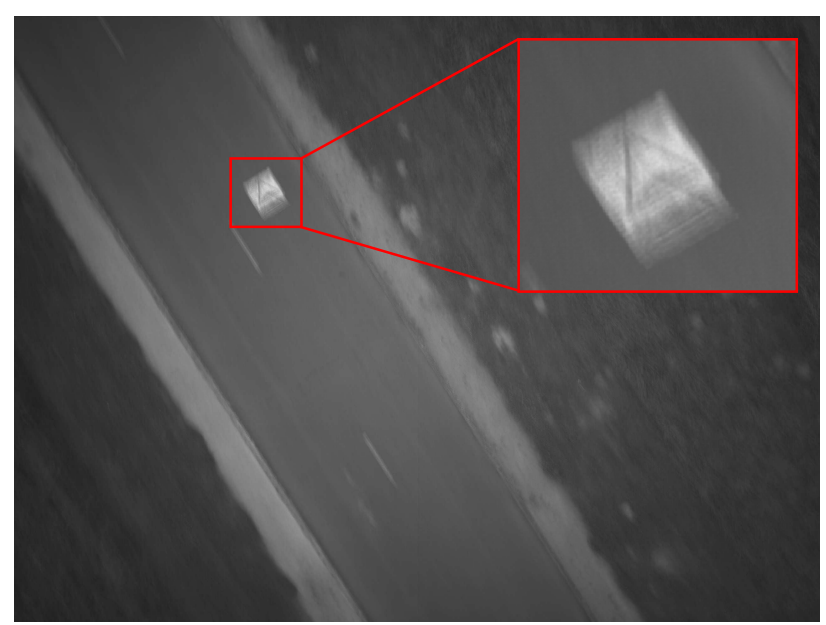

Figure 14. This image is obtained by the average of the ten images. The angular motion between images is 0.9 degree corresponding to 44 pixels.

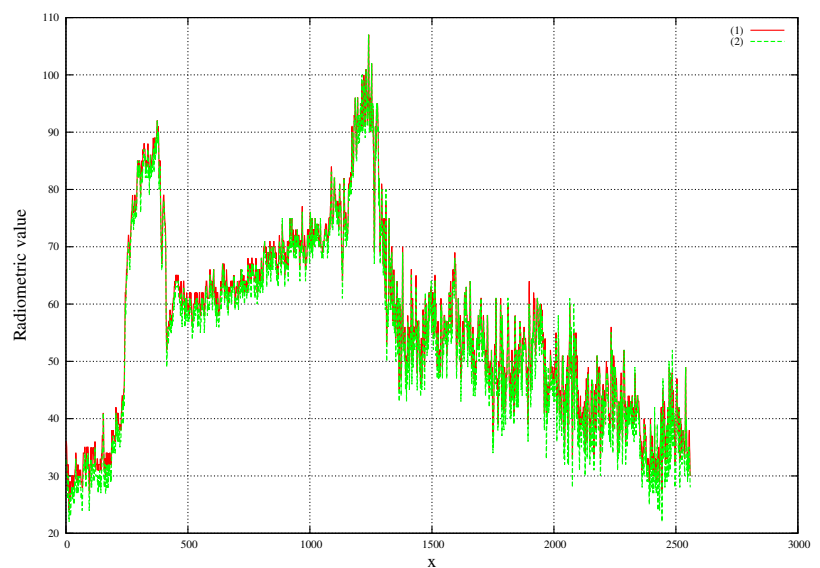

Figure 15. Radiometric profil of the first image (red) and the stacked image (green).

\subsection{CASE OF NON-PURE CAMERA ROTATION MOVE- MENT}

Figure 16 and Figure 17 illustrate a case where the UAV has suffered a X-translation movement. Here, the rotation estimated

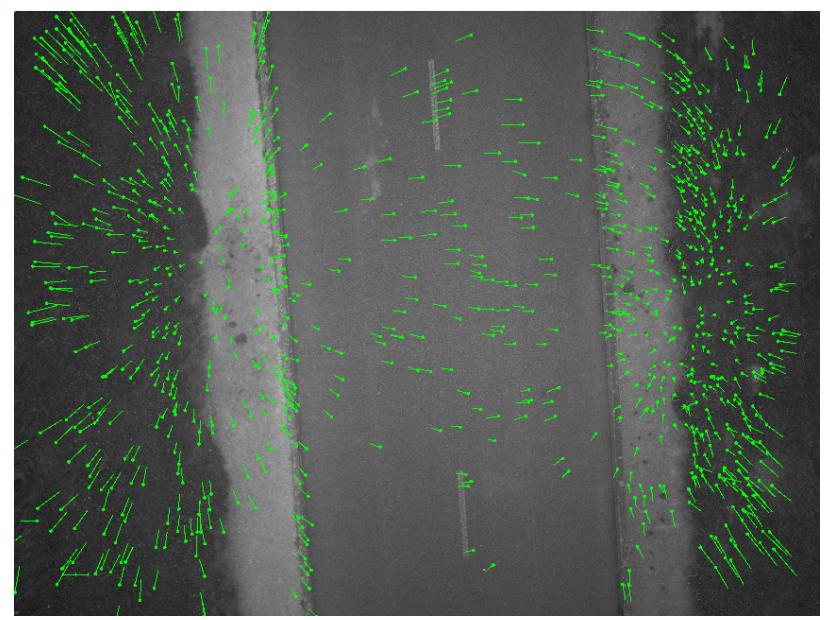

Figure 16. Residus obtained by the $3 D$ rotation estimation between the first image and the $10^{\text {th }}$ image.

with our photogrammetric method is not optimal because the optical center is moving, thus the residual errors are greater than 
expected. This residual movement, which is not compensated for by the rotation, can be absorbed by 2D homography, and Figure

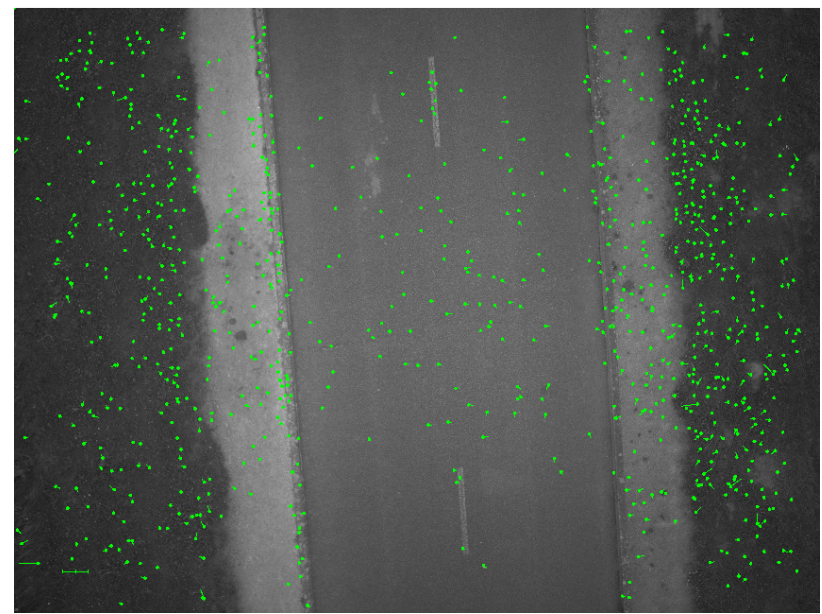

Figure 17. Residus obtained by the 2D homography estimation between the first image and the $10^{\text {th }}$ image.

17 demonstrates that the residual errors in the estimated homography are constant over images and much lower than those for the estimated 3D rotation. This translation movement has been proved by calculating the external orientation of the camera during each acquisition (with MicMac software).

\subsection{QUALITY OF THE STACKED IMAGE OBTAINED BY THE ACCELERATED METHOD}

Figure 18 and Figure 19 demonstrate that the difference (in pixels) according to the two axis of image between the projected point obtained by the precise mapping and the projected point obtained by the bilinear function is continued especially across the borders of blocks in the image.

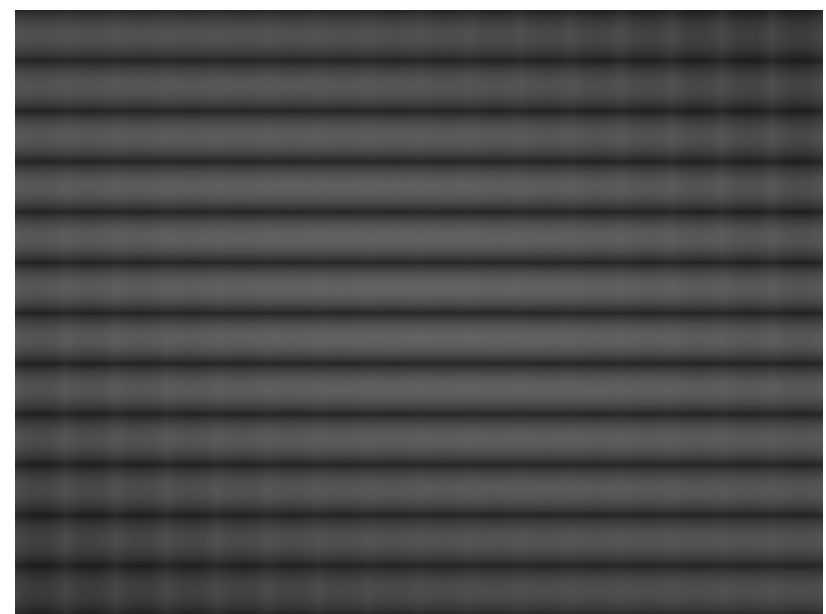

Figure 18. Difference (in pixels) according to x axis for each pixel between the projected point obtained by the precise mapping and the projected point obtained by the bilinear function between the $1^{\text {st }}$ and the $10^{\text {th }}$ image : Dif $f_{\max }=0.0146$.

Additionally, the maximum distances (in pixels) between points projected by the precise mapping and points projected by the bilinear function doesn't exceed the 3 hundredths as shown in Figure 20. From the two stacked images obtained using the two accelerated methods, the two radiometric profiles present a very similar content as presented in Figure 21 that means that the accelerated method doesn't affect the quality of the stacked image.

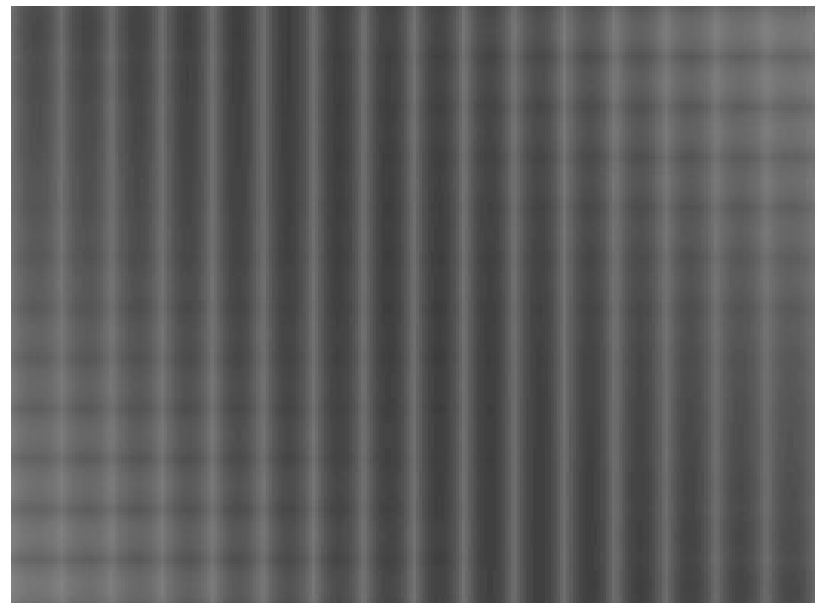

Figure 19. Difference (in pixels) according to y axis of image for each pixel between the projected point obtained by the precise mapping and the projected point obtained by the bilinear function between the $1^{\text {st }}$ and the $10^{\text {th }}$ image : Dif $f_{\max }=0.0162$.

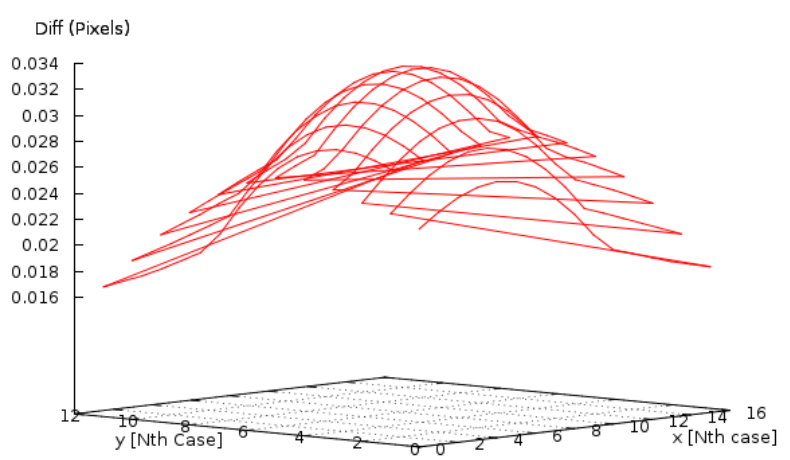

Figure 20. Maximum distance (in pixels) between the point projected by the precise mapping and the point projected by the bilinear function in each block of the grid of the image.

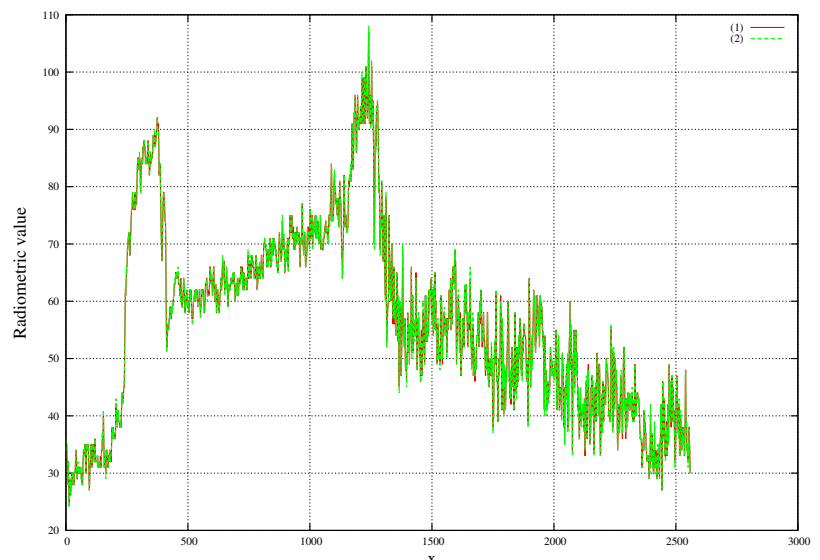

Figure 21. Radiometric profile for : (1) the stacked image obtained by accelerated transformation and bilinear interpolation, (2) the stacked image obtained by accelerated transformation and nearest neighbour.

\section{CONCLUSION}

A real-time image processing stacking algorithm has been presented in this paper. The proposed method was tested with the IGN camera embedded in an UAV, and the experimental results show the efficiency and accuracy of this method when the UAV is quasi-stationary. Images can be improved to a point where the 
blur is almost undetectable even if the movement of the camera is complex. Our method can be applied for images that exceed an angular motion blur of much more than several pixels unlike deconvolution methods (Shah and Schickler, 2012) that have strict limits on the amount of angular motion. IMU data processing is already real-time and can be used for other applications. Other sensors like other synchronized cameras or lidar can benefit from the resulting better quality of the IMU data. As an extension, this work is intended to be used on an airborne prototype imaging system with numerous spectral channels composed of several synchronized IGN cameras for remote sensing applications. We will test this method with very low light levels that are more realistic in the case of narrow spectral bands.

In the future, we will integrate the translation of the UAV in our model using GPS/IMU data, which may later be used to correct translation induced deformation.

\section{ACKNOWLEDGEMENTS}

We thank here IFSTTAR Laboratory and especially J.L. Sorin for the use of their Copter UAV to perform the experiments.

\section{REFERENCES}

Daakir, M., Pierrot-Deseilligny, M., Bosser, P., Pichard, F., Thom, C. and Rabot, Y., 2016. Study of lever-arm effect using embedded photogrammetry and on-board gps receiver on uav for metrological mapping purpose and proposal of a free ground measurements calibration procedure. ISPRS - International Archives of the Photogrammetry, Remote Sensing and Spatial Information Sciences XL-3/W4, pp. 65-70.

Kraft, M., Schmidt, A. and Kasinski, A. J., 2008. High-speed image feature detection using fpga implementation of fast algorithm. In: A. Ranchordas and H. Araújo (eds), VISAPP (1), INSTICC - Institute for Systems and Technologies of Information, Control and Communication, pp. 174-179.

Le Moigne, J., Netanyahu, N. S. and Eastman, R. D. (eds), 2010. Image registration for remote sensing. Cambridge University Press, Cambridge, New York.

Le, X. and Gonzalez, R., 2009. Pattern-based corner detection algorithm. In: Image and Signal Processing and Analysis, 2009. ISPA 2009. Proceedings of 6th International Symposium on, pp. 238-243.

Martin, O., Meynard, C., Pierrot Deseilligny, M., Souchon, J.-P. and Thom, C., 2014. Réalisation d'une caméra photogrammétrique ultralégère et de haute résolution. Colloque Drones et moyens légers aéroportés d'observation, Montpellier, France.

Pena, M., 2012. A Comparative Study of Three Image Matching Algorithms: Sift, Surf, and Fast. BiblioBazaar.

Pierrot Deseilligny, M. and Clery, I., 2011. Apero, an open source bundle adjusment software for automatic calibration and orientation of set of images. ISPRS - International Archives of the Photogrammetry, Remote Sensing and Spatial Information Sciences XXXVIII-5/W16, pp. 269-276.

Rosten, E. and Drummond, T., 2005. Fusing points and lines for high performance tracking. In: Proceedings of the Tenth IEEE International Conference on Computer Vision - Volume 2, ICCV '05, IEEE Computer Society, Washington, DC, USA, pp. 15081515 .
Shah, C. A. and Schickler, W., 2012. Automated blur detection and removal in airborne imaging systems using imu data. ISPRS - International Archives of the Photogrammetry, Remote Sensing and Spatial Information Sciences XXXIX-B1, pp. 321-323.

Tournadre, V., Pierrot-Deseilligny, M. and Faure, P. H., 2015. Uav linear photogrammetry. ISPRS - International Archives of the Photogrammetry, Remote Sensing and Spatial Information Sciences XL-3/W3, pp. 327-333. 